\title{
Addendum: Wnt4 signaling prevents skeletal aging and inflammation by inhibiting nuclear factor- $\kappa \mathrm{B}$
}

\author{
Bo Yu, Jia Chang, Yunsong Liu, Jiong Li, Kareena Kevork, Khalid Al-Hezaimi, Dana T Graves, No-Hee Park \& Cun-Yu Wang \\ Nat. Med. 20, 1009-1017 (2014); published online 10 August 2014; addendum published after print 4 August 2015
}

In the published article, we, the authors, interpreted the data in Figure 4a-e to mean that transgenic expression of Wnt 4 in osteoblasts of mice protects them from age-related bone loss. However, after publication it has been pointed out to us that, at all the ages examined, the transgenic mice had greater trabecular bone mass than control mice. Thus, we have re-examined the data in Figure $4 \mathrm{~b}$ using statistical tests to examine the relative rate of change of bone mass and bone volume/total volume over the four age groups presented, and we find there is no statistical difference for the rate of these parameters between the transgenic and control groups. Thus, our conclusions with respect to this aspect of the study were incorrect, and further we conclude that we placed an improper emphasis on these findings in the title of the paper.

We have also reanalyzed the data in the rest of the paper using more proper statistical tests in several instances. In particular, the standard deviations in Figures 1g, 1h, 2c-e, 3c-e, 4d, 4f, 6d, 6e and Supplementary Figure 6g were reported inappropriately. We used values from each histology image (3-6 images per mouse) as individual data points, instead of the mean values for each mouse, leading to an increased standard deviation. Furthermore, for morphometric and serum analysis in Figures 2, 3, 4g,h, 6 and Supplementary Figure 6, one-way analysis of variance with Tukey's post hoc test should have been used to account for multiple comparisons and adjustments for type I errors. Upon reanalysis, the comparison of osteoclast numbers/bone surface between the wild-type (WT) sham group and the Ob-Wnt4 sham group in Figure 2e, and the comparison of osteoclast surface/bone surface between the WT and Wnt4 groups in Figure 3e lost statistical significance, as was stated in the article.

Although our conclusion about the effect of transgenic expression of Wnt4 in osteoblasts on skeletal aging appears to be incorrect, the above changes regarding our statistical analyses do not alter the conclusions drawn in the manuscript with respect to the effect of Wnt 4 transgenic expression on bone mass compared to non-transgenic mice at static time points, the effect of recombinant Wnt 4 on bone loss in the ovariectomy model nor on the molecular mechanisms for these effects. Nonetheless, we apologize for any confusion these original analyses or conclusions may have caused. 